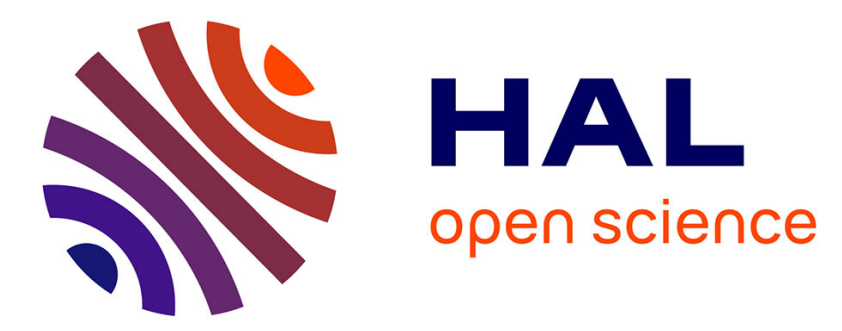

\title{
Science education as a driver of cyberspace technology development
}

Roy D. Pea, Louis M. Gomez, Daniel C. Edelson, Barry J. Fishman, Douglas N. Gordin, D. Kevin O’Neill

\section{> To cite this version:}

Roy D. Pea, Louis M. Gomez, Daniel C. Edelson, Barry J. Fishman, Douglas N. Gordin, et al.. Science education as a driver of cyberspace technology development. K. C. Cohen. Internet links for science education, New York, NY: Plenum Press, pp.189-220, 1997. hal-00190603

\section{HAL Id: hal-00190603 \\ https://telearn.archives-ouvertes.fr/hal-00190603}

Submitted on 23 Nov 2007

HAL is a multi-disciplinary open access archive for the deposit and dissemination of scientific research documents, whether they are published or not. The documents may come from teaching and research institutions in France or abroad, or from public or private research centers.
L'archive ouverte pluridisciplinaire HAL, est destinée au dépôt et à la diffusion de documents scientifiques de niveau recherche, publiés ou non, émanant des établissements d'enseignement et de recherche français ou étrangers, des laboratoires publics ou privés. 


\title{
Science Education as Driver of Cyberspace Technology Development
}

\author{
Roy D. Pea, Louis M. Gomez, Daniel C. Edelson, \\ Barry J. Fishman, Douglas N. Gordin, and \\ D. Kevin $\mathrm{O}^{\prime}$ Neill
}

\section{INTRODUCTION}

Educational applications of networking technologies are becoming increasingly prevalent (National Center for Education Statistics, 1996; Riley et al., 1996). But "applications" are too often treated as infusions of technology into society, not drivers of new technological or research developments. One premise of the Learning through Collaborative Visualization (CoVis) Project challenges that common belief (Pea, 1993). Extending media-rich and highly interactive learning and teaching activities beyond single classrooms makes demanding requirements for new applications. We set out to create "distributed multimedia learning environments" to serve the emerging needs of precollege science education, which highlight learning through guided inquiry and affiliated new roles for teachers (National Research Council, 1996).

Distributed multimedia learning environments using advanced computing and communications technology can better enable precollege science education

Roy D. Pea, SRI International, 333 Ravenswood Avenue, Menlo Park, CA 94025; roypea@unix.sri.com. Louis M. Gomez, Daniel C. Edelson, Barry J. Fishman, Douglas N. Gordin, and D. Kevin O'Neill, Northwestern University, Evanston, IL60204; I-gomez@nwu.edu, edelson@covis.nwu.edu, b-fishman@nwu.edu, gordin@covis.nwu.edu, and oneill@covis.nwu.edu 
to foster learning science by doing science, using the Internet. In our research, we have been investigating the requirements for placing such environments into classrooms and the challenges that must be met for their appropriation. The implementation and sustained use of such technologies on a large scale basis in $\mathrm{K}-12$ school communities is a challenging design problem, related in large part to the complexities of sociotechnical systems.

The CoVis Project has provided a wide-band high-speed computer network and a desktop video conferencing network, structured groupware for collaboratively developing and conducting scientific inquiries, and scientific visualization tools that provide access to wide-ranging data sets on climate, weather, and other global parameters. The development of the CoVis learning environment and its components was guided by a question-centered and collaboration-focused pedagogy that recognizes students and teachers will continue the design process by innovating uses and suggesting revisions to their functionality.

During the 1995-96 school year, the CoVis Project involved over 100 teachers and more than 3000 students, using telecommunications as a critical leveraging technology to develop and evolve through use a wide array of new resources for learning. This testbed is now creating multiple opportunities for learners to engage in open-ended inquiry and new kinds of learning relationships. In essence, the CoVis Project has established a community of invention, which has spent the past 5 years constructing, experiencing, and evolving new kinds of science learning environments. This chapter provides a wide-angle view on the demands of science learning environments and on five CoVis examples of how these needs have led to new cyberspace technology developments.

\section{THE COVis PROJECT}

Our goal has been to create a scalable approach to the establishment of distributed multimedia learning environments for high school and middle school science. We have designed and now provide wide-area broadband services that integrate educationally appropriate scientific visualization tools, newly developed structured hypermedia collaborative workgroup software, desktop videoconferencing and screen sharing, and standard Internet communication tools Why did we take this approach?

Distributed Science Learning: Serving Science Education Needs with Cyberspace Technology Development

The CoVis Project was founded on the premise that classroom science learning should more closely resemble the open-ended, inquiry-based approach of science practice. As we put it in the January 1992 proposal to the National Science Foundation:
We believe that science learning environments should look and act more like the collaborative, connected work environments of scientists. To this end, teachers and students need ways to reduce the complexity of getting access to resources that are inaccessible locally. These resources include human expertise in the form of other teachers, scientists and graduate students in business, industry, and research settings, and other learners. They also include tools, instrumentation, hands-on materials and labs. museum exhibits, and computing and telecommunications infrastructures....

[We] aim: To examine how geographically dispersed teachers, students, and collaborators can integrally and readily use advanced information technologies to facilitate the types of collaboration and communication demanded by project-enhanced science learning.

We argue that applications of advanced technologies provide American educators with critical levers for promoting cognitive apprenticeships in science learning. We aim to build the next generation of infrastructure for new forms of science learning and teaching, and create a national model for the kinds of distributed multimedia science learning environments supportable with the future NREN.... We argue that these new [High Performance Computing and Communications] technologies can provide the backbone for the transitioning process from didactic science teaching to cognitive apprenticeships in project-enhanced science learning.... Students need to learn and do science in context of real problems and with sophisticated tools.

The CoVis Project thus designed a "network testbed" in which such a vision for science educational reform - that takes cognitive, social, technological, and scientific breakthroughs into account-could be concretely implemented and then empirically studied as a community appropriates and evolves its uses. We found it essential to attend to learning and teaching needs, as described below, in the context of technology trends. Our attention to trends in the evolving national information infrastructure when we began in early 1992 led us to client-server distributed network architecture, to scientific visualization as a substantive emphasis, to designs for new collaborative tools for enabling joint work among widely distributed CoVis community participants, and, initially to our use of a public-switched ISDN network. (We later moved to a variety of schemes for Internet connectivity as they became available.) In short, we sought to create a model community of distributed science learning.

Our assessment of learning and teaching needs led us to project-enhanced science learning as a fundamental pedagogy for achieving deeper understanding (e.g., Ruopp et al., 1993). This approach to science education was developed as a combination of several current strands of research in the cognitive sciences of learning. Chief among these is the conception that, long before and also outside of formal education, people have learned through participation in communities of practice (e.g., Lave and Wenger, 199.1). The advantage of such communities, in contrast to the regimens of "delivery-oriented" instruction common today, is that learning is situated with respect to community-based goals and activities in which knowledge is developed and used (Brown et al., 1989; Pea, 1992; Songer, 1995). In the classroom, this advantage may take the form of what Collins et al. (1989) called cognitive apprenticeship, with students 
guided, both by their teachers and by remote mentors, to think about science in many of the fundamental ways that scientists do. In designing CoVis classroom activities, we quickly realized that there were vast distances, both cultural and physical, separating classrooms from scientists. The point of science education is not to take the "little scientist" that Piaget said was in every child and make him or her become an actual scientist. In recognition of this separation, our goal was to design settings in which students could become what Lave and Wenger (1991) have called "legitimate peripheral participants" in communities of science practice.

In the community of science practice, the tools used and the data collected for investigations are critical to the scientists' abilities to formulate, think about, and work on their problems (e.g., Cerf et al., 1993). In designing the CoVis testbed, we sought to take advantage of this reliance by providing students with ways to access the same data the scientists study using tools modeled on those used by scientists. Providing access to these tools and the scientists and other professionals who use them is one of the key design techniques we used to foster a sense of legitimate peripheral participation. There are other recent efforts to bridge the science education-scientific practice "gap," including Global Lab, Project GLOBE, GREEN, EARTHWATCH, and Hands-On Universe, which have developed related models, of "student-scientist" partnerships (see this volume).

\section{Education Is a Demanding Environment}

Computer and communications science and industries make a mistake when they assume that advances in technology stem either from technological innovations and engineering alone, or from generalized theories of human-computer interaction. We know from work this past decade on "user-centered system design" for software systems that it is exceptionally important to fit the tool to the task, even as we seek to invent technology paradigms and applications so that new tasks become possible (e.g.. Greenbaum and Kyng, 1991; Nardi, 1996; Norman and Draper, 1986; Schuler and Namioka, 1993).

Education in particular has suffered because it is often the last application area for advanced technologies. It has typically taken 15 years for computer tools to make their way into classrooms from their advent in military, industrial, and university settings (U.S. Congress Office of Technology Assessment, 1987). Examples include Logo programming, spreadsheets, graphing tools, simulations, and word processing. But education is far too crucial a function for the future of our increasingly global societies to wait in this long line.

In precollege education, children of different ages participate daily in one of the most demanding challenges of a lifetime. They are supposed to devote some 18,000 hours over about 200 seven-hour days a year for 13 years to learn to engage the different abilities of the mind and body, to become literate in the subject matter, skills, and media of their era, and to contribute to society in work, family, and community. Until recently, students' primary setting for doing so was with one or a few teachers, within a few small rooms, not connected to the world even by a telephone.

Education is not only an "application area," but what we will call a high-priority "invention area." Industry could benefit from working with educational professionals and researchers in the learning sciences to understand the market opportunities surrounding the provision of better support to the activities of learning and teaching.

Although teachers have adapted previous "innovations" such as slide projectors, audiorecorders, radio and TV without making substantive change in their teaching (Cuban, 1986; Tyack and Cuban, 1995), there is strong evidence that teachers are recognizing how computer and communications technologies have vast potential to provide new work tools for transforming their educational practices and the learning experiences and outcomes that are possible in fundamental and exciting ways (Fisher et al., 1996; Means, 1994).

The demands and constraints of educational settings can drive the development of very interesting and valuable new technologies and paradigms of computer and telecommunications use in support of human activities. Several years ago, Pea and Gomez (1992) made this argument for the creation of distributed multimedia learning environments that integrate computing and communications to provide students with rich resources for learning accessed in the context of geographically separated learners.

\section{Science Education as Driver of CoVis Technology Developments}

We now provide five examples, in a common framework, for how the demanding needs of science education have led to new cyberspace technology developments in the CoVis Project. A preliminary overview of the CoVis network environment and "testbed" suite of applications will be useful. We began working with six earth and environmental science teachers at two Chicago-area high schools in summer 1992. In fall 1993, these teachers and nearly 300 students began the school year with scientific visualization tools for atmospheric sciences and an asynchronous collaboration environment called the Collaboratory Notebook, both developed by the CoVis Project, as well as desktop video teleconferencing, and a full suite of Internet tools including e-mail, Usenet news, and Gopher. Students in each classroom used these applications running on a group of six Macintosh Quadra workstations connected to a high-speed video and data network. These applications collectively provide a "collaboratory" environment (Lederberg and Uncapher, 1989; Cerf et al., 1993) that couples tools to support communication and collaboration with open-ended scientific inquiry tools. As of November 1996, we are now working with over 40 schools, more than 100 teachers, and over 3000 students who are participants in the CoVis Collaboratory testbed. 
Network Infrastructures for Shared Media Spaces

Context of Problem. K-12 schools have not generally benefited from internetworking technologies. Although there were over 5.8 million computers in use in schools as of 1995 , only $12 \%$ of schools at that time had connections to some kind of wide-area network (U.S. Congress Office of Technology Assessment, 1995), and fewer than half of those schools had connections to even one classroom (Heaviside et al., 1996). As many as $50 \%$ of teachers have little or no experience at all with technology in the classroom (McKinsey \& Co., 1995). As the June 1996 report to the nation from the Office of the U.S. Secretary of Education characterized the current situation:

Computers and information technologies are not part of the way most American students learn. Today's students spend an average of only a few minutes a day using computers for learning. Only 4 percent of schools have a computer for every five students-a ratio sufficient to allow regular use. Only 9 percent of classrooms have connections to the Internet. (Riley et al., 1996)

One major goal of the CoVis Project, which began in 1992 when school internetworking was far less developed than in its 1996 state just described, has been to combine prototype and off-the-shelf applications to create a reliable, networked environment that showcases High Performance Computing and Communications technologies for $\mathrm{K}-12$ learning communities. We intended to circumvent the traditional decade-long "trickle down" of technology from industry to education (Pea and Gomez, 1992) and design a first-rate suite of technology to support new forms of science education.

Educational Needs of Learners and Teachers. As in related industrial work at Bellcore and Xerox PARC, we wished to create rich "media spaces" (Gomez et al., 1994) to support collaborative work and learning among adolescent students, their teachers, and scientist mentors. Research in the learning sciences has indicated that learners and teachers need highly interactive conversational environments around media-rich artifacts to provide common grounds for fostering learning communications (Pea, 1994; Pea and Gomez, 1992).

What Did We Develop? Although our goals have not changed in the 5 years CoVis has been working in schools, our approach to school networking and desktop videoconferencing has changed substantially from the early implementation of CoVis in just two schools to our present day implementation in 43 schools.

In the first phase of CoVis, the network design and implementation was the result of a collaboration between Northwestern, Ameritech, and Bellcore. We selected public-switched Primary Rate Integrated Services Data Network (PRIISDN) as the transport layer for the CoVis network. ISDN bandwidth can be broken up into call channels, and dedicated to different functions, so we were able to create a hybrid, two-function "overlay" network that gives student workstations access to both Ethernet-based packet-switched data services and circuit-switched desktop audio/video conferencing. Each CoVis classroom in the first 2 years of the project contained six networked Macintosh workstations with an accompanying desktop video teleconferencing unit.

At each school, the local Ethernet was bridged through an Ethernet-ISDN bridge and passed through an inverse multiplexer across the ISDN lines to Northwestern's campuswide network. We sought to avoid video call blocking, so each school had enough line capacity to maintain six video calls at once, while still providing $\mathrm{T} 1$-equivalent bandwidth for Internet connectivity. The initial CoVis network layout is shown in Fig. 1.

Desktop video teleconferencing is a critical component of the CoVis testbed. In the first 2 years of the CoVis Project, students used Bellcore's Cruiser application (Fish et al., 1993), allowing them to place point-to-point video teleconferencing calls to other CoVis addressees by selecting an individual's name from a directory. Cruiser is a client application of Touring Machine (Bellcore Information Networking Research Laboratory, 1993), the distributed network software developed by Bellcore for managing heterogeneous media resources (e.g., cameras, microphones, monitors, switch ports, directory serv-

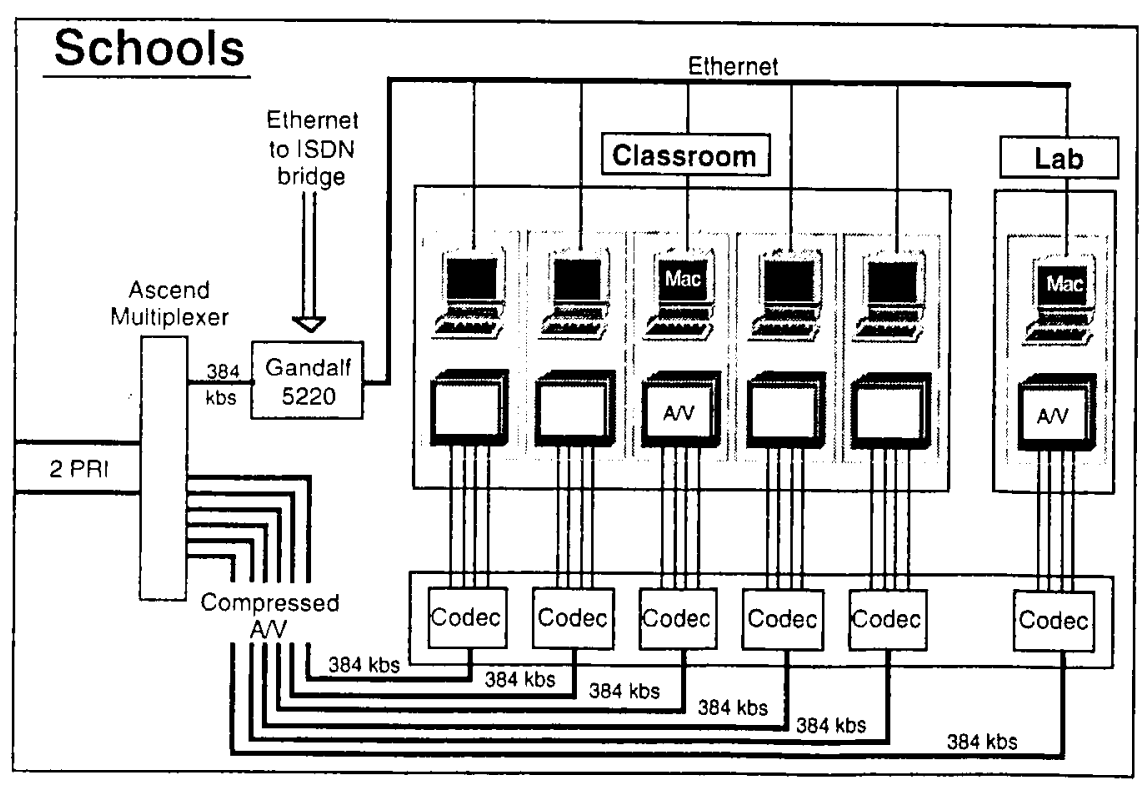

Figure 1. The network configuration for the two Year One CoVis schools, showing both data and video networking. 
ices). It is significant that CoVis Project needs and affiliated Ameritech interests drove the first integration by Bellcore of Touring Machine into an ISDN network. Bellcore's Touring Machine and Cruiser development teams devoted over 2 person-years to the CoVis Project to develop network infrastructure. To our knowledge, CoVis was the first school-based application of ISDN desktop videoconferencing.

In addition to videoconferencing and Internet applications, the CoVis communications suite includes screen sharing. CoVis participants may collaborate synchronously through screen sharing, using Timbuktu (from Farallon Computing), which enables one user to see exactly what appears on the screen of another user, regardless of distance, and users at either end of a screen-sharing session may control the remote computer with their own keyboard and mouse.

In the second phase of the CoVis Project, we did not attempt to devise custom network infrastructures for each participating school. With 41 new schools joining the project, this was not feasible. However, it was also unnecessary, for during the first phase of the CoVis Project a new industry in Internet service providers (ISPs) was quickly becoming established. At the start of the second phase of the CoVis project, it was possible to ask schools to make their own arrangements for networking, at either $56-\mathrm{kb}$ or $\mathrm{T} 1$ levels of connectivity. Instead of network implementers, the CoVis Project served as consultants to these schools, advising them on how to acquire the highest bandwidth at the best price, and how to think about the design of local area networks within their buildings.

During the time that CoVis was experimenting with Cruiser, a new form of videoconferencing tool was starting to emerge that used TCP/IP, or Internet protocols, as its transport mechanism. The premier example of this type of tool was CU-SeeMe, from Cornell University. The primary teclinical advantage of this software was that it enabled extremely low-cost videoconferencing-a school's investment in Internet connectivity also provided their video network. The primary technical limitations for this software in its early stages were a lack of integrated video and audio, and the sometimes complicated process of introducing video into one's computer system. Also, because of the variable bandwidth limitations of the Internet, video quality could be quite low. These tools are "packet-switched" (as opposed to "circuit-switched"), which means that they must share bandwidth with all of the other services in use on the network. Average frame rates for $\mathrm{CU}$-SeeMe were between 5 and 8 frames per second of black and white video, compared with Cruiser's 25-30 frames per second of color video. And, at least initially, you needed to use a telephone to provide audio.

In early 1994, two technological developments made it possible for the CoVis Project to consider CU-SeeMe as a replacement for Cruiser. The first of these was the introduction of synchronized audio in the CU-SeeMe software, using the computer's built-in microphone as the source. The second was the introduction of the Connectix QuickCam, a low-cost digital camera (widely known as the "eyeball" camera for its shape) that required no additional hardware to get video into the computer. These new developments meant that most CoVis schools could now afford to participate in videoconferencing activities, and we discontinued use of Cruiser in favor of CU-SeeMe.

How Did It Work in Use? Our key result from phase one of the CoVis Project was that we were able to establish our ISDN-based network and put it in daily use by approximately 300 people, mainly high school students. The challenge of this effort was to take a collection of technologies, many only demonstrated or tested in small-scale lab situations, and place them into daily service in demanding conditions. Our progress culminated in a stage-by-stage installation in fall 1993 of the CoVis network testbed using public-switched ISDN services. It was used in the schools for approximately 2 years, until it was replaced by more conventional $\mathrm{T} 1$ lines and IP-based videoconferencing using CU-SeeMe (see Desktop Video section for details). During the first year of full implementation, we encountered a range of difficulties stemming from the experimental nature of some of the software, inexperience with novel products and services both within the project and at the commercial service providers, and product and network unreliability. Within 3 months of its initial deployment, however, the data and video network were stable and reliable. How specific applications were used within this networking environment (e.g., visualization, groupware, desktop videoconferencing) will be described in the sections below.

As CoVis moved into its second phase in late 1994, the challenges posed by network development were quite different than in the first phase. Of the 41 new schools joining the CoVis Project at that time, only 5 had prior Internet access. The remaining schools were interested in CoVis in part because we would assist them in obtaining Internet access, and in part because we provided schools with a substantial curricular context for justifying Internet access. The 36 schools that required Internet access all planned to obtain networks during the 1994-95 school year. However, only about half of these schools were actually able to complete their networks during this time period. There were two types of difficulty: First, many schools encountered political difficulty in obtaining permission to install networks from local school boards, related in part to concerns about pornography and the Internet raised by popular press articles during the summer of 1995. A second difficulty was unanticipated physical barriers within school buildings, including asbestos problems, outdated electrical wiring, and lack of adequate security for computers and network hardware. Finally, many schools simply had inadequate technical staff for dealing with unforeseen implementation issues that CoVis staff were unable to resolve from a distance. These issues were particularly challenging for the many urban schools we are working with in this scaled-up testbed, which were among the last to gain network access. All CoVis schools were finally wired by the Fall of 1996, nearly two full years later than many anticipated gaining network access. Among the 
43 CoVis schools, 18 have established Internet connectivity with dedicated $56-\mathrm{kb}$ networks, and the remaining 25 have dedicated T I networks.

What Does This Tell Us about Design Both Generally and Specifically? CoVis experiments with ISDN networks during phase one taught us valuable lessons about the complexities of bringing networks into schools. Although the specific configuration of our ISDN network was not carried through into the second phase of the project, the general lesson to "expect the unexpected" in terms of system start-up problems remained relevant. Among the most important resources for schools to have is reliable technical support in the building. This is necessary not only for the design of networks, but also for their continued maintenance.

What Challenges Remain? Although we now have 43 schools up and running as part of the CoVis Collaboratory testbed, it is still not routine for schools to have Internet networks installed. This is the result of a mixture of technical and social issues. Schools need budgetary support to hire qualified network specialists. Internet providers need to devise pricing structures for school Internet connectivity that fit within the complex budgetary structures of schools. And projects like CoVis need to continue developing compelling activity frameworks to make these Internet connections worthwhile investments for schools.

\section{Adapting Scientific Visualization Tools for Learners}

Context of Problem. When we started our work on scientific visualization for precollege education in early 1992, we were impressed with the utility of general-purpose visualization environments for the scientific research community. Scientists were using visualization to find patterns in complex data using the distinctive strengths of the human visual system (Brodie et al., 1992; Wolff and Yaeger, 1993). Demonstrating support for this method of research were the standardization of data formats and distribution of visualization packages. Science teachers and students could benefit from similar means if they were helped to appropriate and "redesign" visualization for their needs (Gordin and Pea, 1995).

In studying science, students are commonly confused by the abstractions, formalisms, and quantitative terms of equation-based data representations. By taking advantage of the powerful capabilities of human visual perception, scientific visualization offers a new route to scientific understanding, and the possibility of reaching students traditionally ill-served. Scientific visualization also offers the possibility of opening up new domains for study that have been considered too complex for students because of their heavy reliance on formulas and abstract representations. Similarly, scientific visualizations can give students the ability to conduct data-driven investigations in areas to which they previously only had access to broad overviews (e.g., global climate data; planetary biomass distribution; ocean temperature).

We had spent considerable time in science classrooms, and knew from previous work on science educational software that a vast gap existed between what students understood and what they would need to know to use such tools effectively in support of science learning.

Educational Needs of Learners and Teachers. Substantial revisions were necessary to make scientific visualization technologies usable for precollege science students (Gordin and Pea, 1995). Typically, scientific visualization software, such as Spyglass's Transform, provide powerful general purpose means to render data as images. For example, Transform can be used to render temperature and precipitation values as a colored image where the temperature is shown through color and precipitation through contours. The data sets for scientific inquiries like these are often so well known by the scientists who use them that they do not even bother to label the variables represented. The operations in these visualization systems are extensive, as they are designed to cover the common operations of many domains. Scientists typically do not even notice all of the irrelevant or nonsensical operations that are allowed (e.g., subtracting temperature from precipitation). Instead, their actions are guided by the underlying semantics of the quantities they are manipulating that lead them to enact well structured idioms of operations. In this way, scientists demonstrate shared tacit knowledge about the data sets that students lack. For example, scientists are keenly aware of the distortions that spatial projections introduce and factor them into their analyses. Yet students are often unaware of the misleading properties of these projections nor how to render the data so as to compensate for their effects.

What Did We Develop? To adapt scientific visualization for the classroom we "knowledge engineered" atmospheric scientists and reified their analysis techniques into our new visualization tools for learners. We sought to rapidly prototype these new learning environments by building interfaces "in front" of existing scientific visualization tools, thereby creating learner-appropriate interfaces to these tools' already impressive functionalities. These "front-ends" provided supports for novices to learn the techniques and idioms of visualization in a structured way. We defined a four-step methodology for adapting the scientists' visualization tools for education:

1. Investigate science practice. Scientists are observed using visualization tools and data sets for a specific domain, with the goal of eliciting the sorts of questions the visualization tools and data sets can be used to investigate for their area, and how the tools are employed during 
inquiry. A crucial step is choosing a set of highly interrelated data sets within which students can investigate relationships, thereby promoting the development of causal accounts on their part for the domains under study.

2. Identify tacit knowledge used in science practice and make it explicit within our visualization tools. We seek to articulate the tacit knowledge scientists employ when using visualization tools, e.g., scientific principles, understanding of the limitations of data resulting from collection processes and underlying models, and common means used to probe the data for patterns or anomalies. This knowledge is then made explicit in the software interface to assist students in pursuing meaningful questions.

3. Motivate visualization in terms of students'interests, experiences, and conceptions. For example, we often begin students' use of visualization by asking them to draw a visualization of any quantity they choose. The often striking drawings students produce demonstrate visualization as a new expressive medium that students have used (see Fig. 2) to render widely ranging quantities (e.g., unemployment rate, biomes, and use of video games). Similarly, we have designed activities that focus on phenomena familiar to students. For example, using visualizations of incoming sunlight and temperature we problematize the changing of the seasons by identifying apparent anomalies in the observed patterns. Through these steps, we intend visualization to become a tool ready-to-hand for students to investigate problems that matter to them.

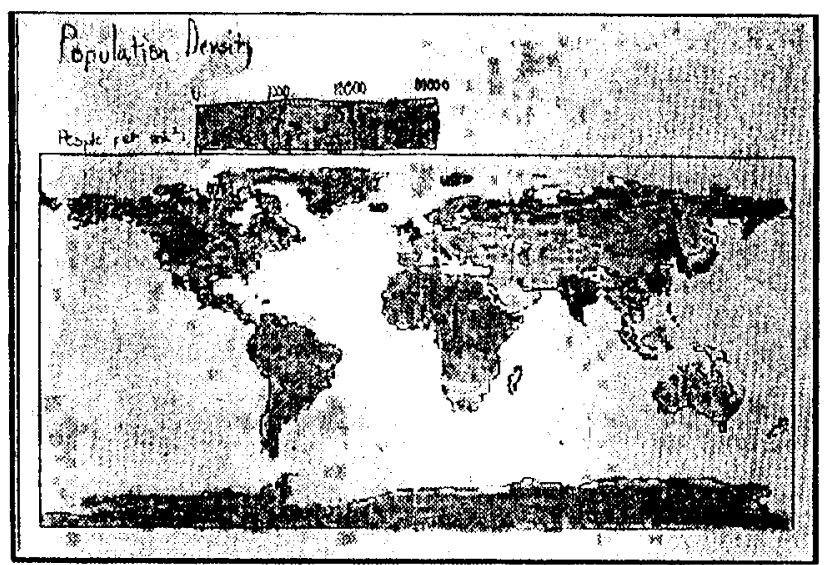

Figure 2. Student hand-drawn visualization showing global population.
4. Refine the visualization tools and activities in response to formative evaluations. Through a combination of observation and direct user feedback, we evaluate the patterns of use that emerge and iteratively redesign the software and activities as needed.

We developed four visualization environments using this process. These visualization environments primarily focus on atmospheric data, although the last also includes human geographic and environmental data sets. These visualization environments are called:

1. Climate Visualizer (Gordin et al., 1994; Gordin \& Pea, 1995)

2. Weather Visualizer (Fishman and D'Amico, 1994; Ramamurthy et al., 1994, 1996)

3. Greenhouse Effect Visualizer (Gordin et al., 1995)

4. Climate Watcher (Gordin et al., 1996a,c)

The first two of these are built on top of a scientific visualization tool used by researchers (e.g., Transform from Spyglass, Inc. for the Climate Visualizer, and WxMap, at the University of Illinois, Urbana-Champaign, for the Weather Visualizer; Ramamurthy et al., 1994). The third uses the World Wide Web to provide visualizations and offers users the ability to query the values of specific points and inspect the visualizations at varied resolutions. The fourth is a stand-alone Macintosh application with a wide range of data sets and many means for rendering and creating visualizations. The increased development costs for this last visualizer were justified by the need to consolidate the findings of prior prototypes (especially the Climate Visualizer and Greenhouse Effect Visualizer). This process illustrates an important way that we have engaged in formative research. Initially rapid prototypes were crafted based on interviews with scientists and other experts, then these prototypes were distributed to teachers and students who aided us in refining the software and collaboratively constructing activities. Only after the design had been refined through use and the creation of productive activities were our designs set into more robust and stable software.

How Did It Work in Use? During 1993-94 and 1994-95, students in the classrooms of the CoVis testbed used the Climate Visualizer and Weather Visualizer in their science projects. Although they found the tools comprehensible and the visualizations useful to offer new insights into several scientific phenomena they were seldom used (e.g., McGee and Pea, 1994; McGee, 1996). This was primarily related to the lack of structured activities that would provide teachers and students with an understanding of their appropriate contexts for use. As a result, teachers perceived relatively narrow curriculum scopes in which these specific scientific visualization programs fit (McGee, 1995), and students often lacked the ability to develop meaningful uses of these investigative tools on their own. 
To address this issue and the broader need to provide a common fulcrum on which to leverage the changes CoVis was bringing to bear, a set of global warming curriculum activities and resources was created and implemented in 24 schools in 1995-96 (Gordin et al., 1996a,b). An integral part of this curriculum were visualization activities where students created visualizations on paper and on Styrofoam models, investigated the causes of the seasons, and analyzed the Earth's energy balance. These activities provided a huge impetus for schools to use visualization and many schools engaged in these activities. However, the style of use ranged widely. At some schools students genuinely engaged difficult issues of representation and meaning by investigating how the data had been collected and the causes of the visual patterns they observed. At other schools the visualizations were merely used as props by teachers to illustrate concepts. In these cases, visualizations became merely another set of color plates added to their textbooks.

What Does This Tell Us about Design Both Generally and Specifically? The iterative and collaborative nature of these designs, which moved technology from the scientists' laboratory to the classroom, requires rapid prototyping to accommodate the multiple cycles of formative research. We successfully built several prototypes by "front-ending" scientific tools with pre-college-age appropriate interfaces, thereby creating a high-end scientific workbench for them. This was an important achievement for us and for our industry partner Spyglass who foresaw a much vaster audience and market for their work-including children with little background in science-thereby opening them to an entirely unexpected market from their perspective.

During this formative process we came to view scientific visualization as a new classroom medium that must allow for student expression and interpretation. Initially, we provided teachers and students with scientists' data and representations and asked them to use them in ways that were analogous to scientists. Over time, we came instead to ask how teachers and students can "play" with visualizations, that is, craft new visualizations and assimilate the medium to their own interests and concerns. This shift does not mean we gave up on the utility of scientists' data or the importance of engaging students in the problems and practices of scientists. Rather, we saw the need for a developmental progression that moved through the stage where visualization is used to serve teachers and students existing concerns before it can serve as a vehicle to introduce new concerns. The need for this step was most palpable when we worked with younger students who had little patience with constructing verbal explanations for complex images, but were excited by the possibilities of crafting colorful images depicting their views of the world. In their images students often depicted human-centered quantities (e.g., population) leading us to add human geographic data to our visualizers and to realize that investigating human spatial patterns can give students a powerful steer on the road toward visualization.
What Challenges Remain? Our success in introducing visualization to science education rests largely on our teachers seeing advantages in using it. In particular, major challenges lie in adapting teacher pedagogy and curricular scope. When teaching is defined in terms of didactic lectures and structured laboratories, there is little room for the open-ended inquiry we are providing tools to support. Similarly, when the curriculum is scoped as covering a long list of topics listed in a textbook's table of contents, there is little room for project study. We believe that "weather" and "climate" and "greenhouse effect" need not be set topics that last for a set number of weeks in the earth sciences curriculum, but topics that involve such interesting, diverse, and complex phenomena that they could be investigated in projects throughout the school year in diverse classes. But to do so required too large a change in teaching philosophy and curriculum theory for most CoVis teachers. On the other hand, it would not have been practical for the CoVis team to conduct the ethnographic observations and interviews that were integral to the design of our knowledge-scaffolding front-ends to the visualization software and data sets for each and every one of the other cognate fields - astronomy, geology, oceanography--which the teachers sought to "cover" in their teaching of earth systems science.

An additional challenge is the development of activities that motivate teachers' initial usage of visualization. So far we have primarily studied how to ignite students' interests in visualization, especially by having them construct their own visualization and engaging problems with which they have experience. Similarly, we must better understand how to meet teachers' existing goals with our visualization environments.

\section{A Groupware Application for Systematic Science Inquiry}

Context of Problem. Early approaches to the use of technology in education were based on a transmission model of instruction, in which the technology (e.g., film and broadcast media) was used to transmit instruction in a more engaging fashion and to larger numbers of students. Distance education inherits this tradition when it uses phone lines, satellite links, and microwave to transmit static knowledge to wider audiences, with minimal opportunities for highly interactive conversations with instructors or learners (Pea and Gomez, 1992).

With the advent of the personal computer, technology took on new roles for learning, including providing responsive environments such as those described in the previous section that support active learning through investigation. However, in the workplace and other environments, computers have also shown great value in supporting communication and collaboration, as we see with e-mail and in groupware tools such as Lotus Notes. Increasingly, technology has assisted in broadening the form that collaboration takes to include not just discussion, but the sharing of artifacts and cooperative work across time and 
distance in shared "media spaces," Technologies with similar emphases can play a revolutionary role in supporting new forms of learning conversations in educational settings (Edelson et al., 1995b; Pea, 1994).

It has become a commonplace for cognitively guided research in science education to document how ineffective lecture- and demonstration-centered pedagogy is at securing student's understanding of subject matter (Linn et al., 1995). Learning that builds from students' questions, and engages the knowledge that they have, is characterized as a "constructivist approach" to learning (e.g., Hawkins and Pea, 1987; Papert, 1994; Tobin, 1993). Projects in the sciences can build from students' questions, and when well guided by mentors, can provide motivating and effective contexts for the acquisition of research skills and scientific understanding (Ruopp et al., 1993). With this in mind, we set out to create a groupware application to foster student learning and systematic science inquiry through project-oriented learning.

Educational Needs of Learners and Teachers. When teachers seek to integrate open-ended science projects into classroom life, they find it difficult to maintain records of students' inquiries and monitor their progress on investiga tions. We expected that well-tailored software could provide significant support for these efforts, especially in a learning environment where many of the artifacts that students and teachers would want to share in the course of their work might be electronic.

What Did We Develop? To meet this challenge, we created a wide-area network-based hypermedia "Collaboratory Notebook." This software was built with an Oracle database server and TCP/IP client software as a foundation (Edelson and O'Neill, 1994), and many classroom-specific customizations on top. The software uses a unique set of inquiry-oriented hypermedia link types for all notebook entries. These link types were intended to "scaffold" students" processes of scientific inquiry, and enable distributed workgroups to more clearly define and conduct science projects in a stepwise (though iterative) fashion.

Within its hypermedia notebooks, the Collaboratory Notebook enables students to record text, tables, graphics, sound, video, and animations throughout their work. These products can then be seamlessly shared with others on the network. With these capabilities in hand, project groups may be composed of students within schools, across schools, and can involve adults as mentors, including teachers, scientists, science graduate students, and science education researchers. Project workgroups are enabled to negotiate project topics with one another and their teachers, and $\log$ observations from scientific visualization tools and hands-on investigations as they complete them. If students' records of their project work are reasonably complete, these can provide teachers and remote mentors with a valuable window into students' inquiry processes and activities.
In an example of how we envisioned the Collaboratory Notebook being used, a group of students would begin by developing an idea for an inquiry, negotiating its details with their teacher, and recording questions and hypotheses electronically. This would be followed by a plan for pursuing their inquiry. A teacher or other mentors located at a distance could, at their convenience, read the students' questions, hypotheses, and plans, and add comments to help them focus their efforts or to alert them to possible stumbling blocks or useful resources. Students would not need to be tied to one another, could go on to engage in separate research activities and individually record these for the others to view at a later time. Students could also electronically exchange questions and comments on their findings, draw conclusions, or initiate further research.

How Did It Work in Use? Beginning in the 1993-94 school year, teachers and students used the Collaboratory Notebook in a variety of ways to support experimental classroom activities. In one case, a teacher conducted a weather prediction activity using the Collaboratory Notebook, in which groups of students recorded predictions for the following day's weather and evidence to support those predictions. Students then had the opportunity to view and comment on the predictions of other groups within the Notebook software. The following day, the predictions were reexamined and discussed in the light of firsthand observations of the weather.

Other, less successful activities planned by CoVis teachers involved students recording questions and progress in the Collaboratory Notebook during more traditional classroom research projects. In these projects, students used few resources beyond standard textbooks and reference materials, and had no audience for their work besides their teacher and the class itself. These activities provided little motivation for students to use groupware, and they sometimes expressed their frustration at this to both the teachers and the researchers.

The Collaboratory Notebook was intended for an environment where access to computing resources was not expected to be a limiting factor. In fact, given access generally to only six workstations in each classroom of 25 students or more (and the slow rate at which many high school students type), limitations on computing resources became a serious constraint on curriculum planning. Accordingly, many projects were developed with only minimal use of the Collaboratory Notebook (Edelson et al., 1995), and some teachers elected not to use the software at all.

One reason why these outcomes are disappointing is that many projects conducted in classes throughout the CoVis testbed could have benefited from the mutual student-to-student influence that the Collaboratory Notebook supports. However, students in each classroom were generally unaware of one another's efforts (O'Neill et al., 1995), and cross-school collaborations rarely transpired. One distant collaboration using the software occurred when CoVis teachers and staff arranged for mentors from the Atmospheric Sciences Department at the 
University of Illinois and the Exploratorium Museum in San Francisco to advise students on their investigations. However, without this coordination effort, the cross-school work that was a key target of the software proved infrequent.

What Does This Tell Us about Design Generally and Specifically? The scaffolding link types in the Collaboratory Notebook were quite attractive to some teachers. In these classrooms they were used to reinforce the teacher's guidance of students in their project work, and aided teachers in their efforts to monitor and shape student progress. Without a classroom culture to give them meaning, however, the link types seem to have had no magic of their own. Heeding the structure of the work tasks in classroom science projects, rather than using off-the-shelf software with untyped links, seems to have paid off for us; but only where teachers were ready to take advantage of and reinforce the software's design.

What Challenges Remain? We would like to see much more integral use of the Collaboratory Notebook in science learning in and out of the classroom. Such use would require several major changes to existing teaching and learning practice, however. One of these is the development of classroom cultures where presentation, commentary on, and revision of projects is more common. Our software is of the greatest use in supporting the revision of project work through helpful, critical commentary by partners distributed across space and/or time. Currently, however, most of the project work we observe is a one-time affair, and does not involve remote partners because of the management effort this requires. Some of our current research focuses on the development of network resources to ease the coordination effort associated with distant mentoring (O'Neill et al., 1996).

Another challenge to the more integral use of the Collaboratory Notebook relates to the many projects under way in the CoVis testbed which have relevance to one another, yet do not find their way into student and teacher discussions (on-line or otherwise). For example, tens of earthquake and hurricane projects are conducted every year by CoVis students, yet these rarely build on each other's findings. For our software to reach its maximum usefulness, this routine would have to change. This aim would require establishing a critical mass of classrooms throughout our network with students wishing to do projects together, and sufficient experience among their teachers to establish common standards for assessing the quality of the learning and research produced by these students.

A third and final change that would increase the use of software like ours would be the availability of a computer and network connection to every student and her teacher, both during school and, ideally, after school. The importance of making a computer and network connection available after school hours is especially great for teachers, for whom it is necessary to review student work at night and at home. An alternative in this vein would be to develop designs for groupware applications that would encourage use without each person having personal, on-demand access to the network.

\section{Desktop Videoconferencing for Classroom Use}

Context of Problem. Considerable enthusiasm has been developing in the industrial community, and in the "new media" marketplace for video-to-thedesktop. The industry focus has been on supporting formal videoconferencing participation by groups who do not need to go to a special facility, or on supporting the informal communications, much like running into a colleague in a coffee area, that tend to enhance the chances that such colleagues will engage in collaborative work (Fish et al., 1993). There have been complex technological and social design issues. Technologies for desktop videoconferencing have been expensive primarily because of codec costs, and sustaining acceptable frame-rate performance for person-person communication needs over either LANs or WANs has provided a demanding environment for engineering. On the social side, the crafting of software and communication protocols for launching and managing desktop videoconference calls to support human communicative needs at work has taken considerable effort. When we began in early 1992, videoconferencing to the desktop in the classroom was virtually nonexistent, although many examples existed of "distance learning" uses of classroom-based videoconferencing (Pea and Gomez, 1992).

Educational Needs of Learners and Teachers. Our expectation was that there were a range of likely needs of the activities that go on in a project-enhanced science classroom which desktop videoconferencing could fulfill. First, we conjectured that a teacher's use of initial resource materials to motivate student interest in a science topic from which projects could be developed, such as videotapes, would carry over to a use of remote visits to interesting video sources, such as exhibits at the Exploratorium Science Museum (a CoVis partner). Second, we conjectured that students would wish to conduct some of their collaborative work across schools with the additional media channel of desktop video, to enhance the communicative feedback possible as they worked to further their project activities. And third, we conjectured that being able to create a distributed audience for the presentation and discussion of project results would be an appealing use of the desktop video medium.

What Did We Develop? We made it possible, with extensive engineering and redesign work from Bellcore and Ameritech described earlier, for students to use the Cruiser application to place calls to other CoVis addressees, including other classrooms and Northwestern researchers (see Fig. 3). Late in the 1993-94 school year, Cruiser installations at the Exploratorium Science Museum in San 


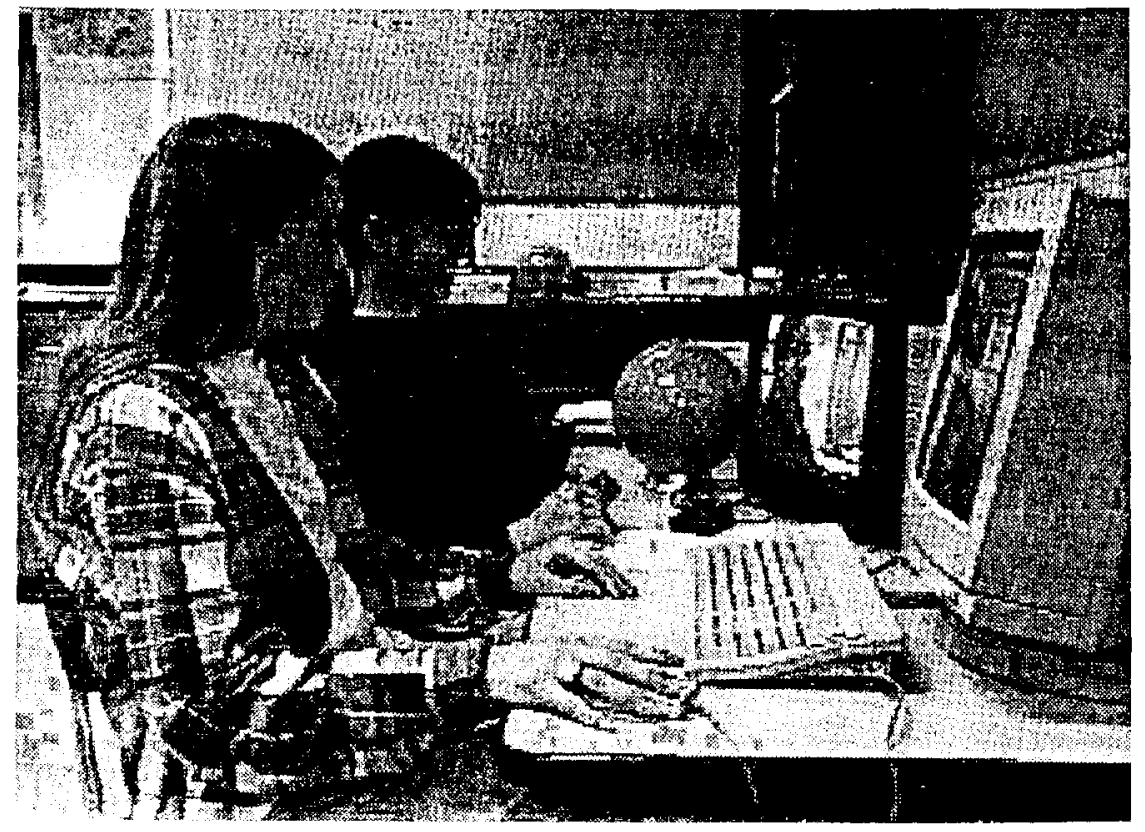

Figure 3. High school students using Cruiser and screen sharing of visualizations.

Francisco and at the Department of Atmospheric Sciences at the University of Illinois, Urbana-Champaign made possible virtual field trips and interactive weather briefings.

How Did It Work in Use? In our initial discussions with teachers prior to the CoVis "launch" during the 1993-94 school year, Cruiser was viewed with great interest. This was understandable, as videoconferencing clearly offered the most immediately comprehensible and dramatic changes of all of the new CoVis technologies being introduced to their classrooms. Scientific visualization and collaborative groupware took longer to comprehend.

As researchers, we were also very interested in the success of Cruiser because it offered a real-time bridge between the communities of schools and scientists. Furthermore, Cruiser also required a sizable investment of funds to become operational, including ISDN telecommunications charges, hardware for video compression and decompression, cameras, monitors, and personnel to help set it up, debug it, and keep it running. Cruiser was technically available in CoVis classrooms beginning in January 1994.

Yet even with the extra emphasis placed on it in the overall design of CoVis classrooms, Cruiser was hardly ever used in daily classroom life. Tracking of the use of all computer-mediated computer tools during the 1994-95 academic year showed that whereas students sent over 10,000 e-mail messages, posted over 1400 Usenet news articles, and created over 1100 pages in the Collaboratory Notebook, there were only 32 Cruiser calls placed (Fishman, 1996). Surprisingly, it was used primarily for troubleshooting pedagogy and software use between teachers and Northwestern research staff, not between classrooms in cross-school collaborative projects as anticipated. How can the extremely low use of Cruiser be explained?

Many of the major difficulties were sociocultural in nature, not technical. Although teachers readily accepted the challenges of creating highly collaborative and interactive environments supported by asynchronous tools (e.g., e-mail, Usenet news. CoVis Collaboratory Notebook), they were more reluctant to include synchronous communication tools in these environments. Reasons for this that emerged over time included:

1. Social context violations. Serious problems emerged with the presumed ease of translating the "office drop-in" model of use for Cruiser videoconferencing in a corporate work environment to classrooms in schools. In the workplace, Cruiser was used to create extended workspaces, helping to foster the kind of informal interaction that people have while "cruising" the hallways of their office space (Fish et al., 1993). Teachers did not like the thought that somebody could interrupt their classroom activity without warning. So Cruiser stations in classrooms were as a matter of practice not "left on" as they were in the offices of Bellcore workers.

2. Issues of coordination with distant partners. One problem that surfaced had to do with the tight windows of available time that any single classroom could be on-line. Whereas in the workplace environment one might reasonably expect to find someone at her desk at any time during the day, in the classroom environment particular students are only typically present for $\mathbf{4 0}$ minutes at a time. Although it may be possible for a scientist mentor to adjust her schedule to be available during a particular class period, the problem becomes much worse when two teachers or students at different schools wish to communicate. As school time is rigidly structured, special arrangements must be made to facilitate interaction between two parties who do not happen to have class during the same hour.

3. Adolescents and school culture. Teachers were worried that adolescents would use desktop videoconferencing as a medium for socializing and ignore their project work, so they were leery to incorporate videoconferencing into project activities or their requirements. This limitation in access for the students meant that it was not used in their investigative work as we had anticipated. It violated classroom communicative practices. 
Furthermore, few cross-school projects developed, as it turned out that teachers did not have a strong enough motivation in favor of cross-school collaboration by students to overcome the challenges that such collaboration presented. For example, the teachers had never before needed to negotiate standards for assessing student work on projects across schools. Without common grounds for assessment, they were less likely to encourage cross-school student collaborations. Reflecting this difficulty, video conferencing was not used within cross-school collaborations. The same limitation prevented the expected use of Cruiser to extend the audience for presentations of students' projects.

4. Lack of critical mass of users. Another coordination challenge was presented by the fact that Cruiser did not support communication between CoVis students and relevant outside populations. Although there were some scientists using Cruiser that students could communicate with (at the Exploratorium and UIUC), the vast majority of students established connections to outside mentors and others using e-mail, which was more widely available and allowed for students and outsiders to communicate on their own, nonsynchronized schedules. Thus, Cruiser was also prevented from flourishing because of the lack of what media theorists have called "critical mass" (Markus, 1987).

The switch to CU-SeeMe helped solve some of the problems described above, but not all of them. During the 1995-96 academic year, videoconferencing using $\mathrm{CU}$-SeeMe began to take root as part of regular CoVis activities. Although the cultural issues of synchronous tools were still present, the associated cost and complexity issues were greatly reduced, and the critical mass problems were minimized. We worked to ameliorate the cultural problems caused by synchronous communication through scaffolding and structuring its use in interschool curricular activities.

One example of videoconferencing activity embedded within a larger curricular context was a model summit on global climate change, held as a culminating activity for a month-long set of activities on climate change (Gordin et al., 1996b).

We have also been able to use desktop videoconferencing on CoVis workstations to provide live daily "interactive weather briefings" from atmospheric researchers (faculty and students) at the UIUC Weather Room. Such briefings can illustrate, through interpretation and analysis of weather charts, satellite and radar animations, and forecast products, key concepts that will enable a student to conceptualize the structure and dynamics of the atmosphere. CoVis students participate in real-time discussions of weather processes, collaboratively interact with scientists around shared data involving atmospheric, kinematic, and dynamic processes depicted on weather charts.
A third type of activity was the "virtual field trip" between various CoVis schools and San Francisco's Exploratorium Science Museum. Using a backpack equipped with a video camera and a remote link to the Internet, museum staff in San Francisco can lead live interactive tours of various exhibits (e.g., investigating the weather section, or exhibits on erosion), as directed by students using CU-SeeMe.

A fourth type of use, unanticipated by the designers at the start of the school year, was that of CU-SeeMe to supplement parent-teacher meetings and informational sessions. This allowed staff at Northwestern to be invited "into" classrooms to answer questions about the various activities students were engaged in as part of CoVis. This combination of ways that CU-SeeMe based videoconferencing has been employed by CoVis teachers and students is an important indicator of its successfully taking root in classroom culture, especially when compared with Cruiser.

What Does This Tell Us about Design Both Generally and Specifically? Our experiences with Cruiser taught us that sociocultural barriers to use of synchronous tools in the classroom are more relevant to design of videoconferencing tools than pure technological issues, and also that cost and complexity need to be eliminated nearly completely if videoconferencing is to stand a chance at adoption. The combination of these difficulties and a lack of critical mass made it highly unlikely that Cruiser would be adopted by CoVis students and teachers. When the CoVis Project switched to CU-SeeMe, we were able to focus not on the technology but on the design of activity with embedded videoconferencing to overcome some of the social barriers.

Most importantly, the "office worker" paradigm of informal videoconferencing to the desktop does not transfer "whole cloth" to the classroom (Fishman, 1996). New design must acknowledge the different goals and social arrangements present in classroom environments than in the workplace. The social conditions for collaboration assumed to be supported by videoconferencing must be in place before the tool will be seen as serving useful functions. Furthermore, the user interfaces to videoconferencing applications for the classroom must be specially designed. Just as the Cruiser application we used was crafted with the realities of the white-collar office in mind, we have to articulate the design constraints from the classroom that should shape informal videoconferencing for teaching and learning.

What Challenges Remain? An inherent weakness of Internet-based videoconferencing systems such as CU-SeeMe is that they take up so much bandwidth that it becomes impossible to simultaneously use screen-sharing software such as Timbuktu. This poses a serious design challenge for CoVis pedagogy, which has as its goal the creation of seamless rich media environments to support collaborative learning at a distance. One possible solution to this 
problem may lie in the construction of Web sites where meeting participants can store the work to be discussed in advance. Another solution is being developed by Apple Computer, in its QuickTime Conferencing (QTC) software. QTC is similar to CU-SeeMe, except that it includes a shared whiteboard facility that can be used to exchange screen shots. Our experiences to date with both improving software and growing network capacity leave us optimistic about the potential for Internet-based videoconferencing as a regular feature of science education in the future.

We are finding a strong potential in uses of CoVis testbed videoconferencing in support of scheduled events that can be drawn on by teachers as dynamic classroom resources. These events-interactive weather briefings, and virtual field trips to a science museum-have allowed students and teachers to interact remotely with scientific experts in informal and formal activities. How to effectively scale network-accessible video events is an obvious challenge. Here, too, designs must be evolved for how teachers and children will make use of these events for classroom purposes. As there are no preexisting models of applications like these, a certain amount of experience-informed iteration will be necessary. Much work remains to change the ways that schools think about the division of time during the day to help make synchronous communication tools such as desktop videoconferencing more generally applicable.

\section{An Educationally Appropriate World Wide Web Resource}

Context of Problem. Exponential growth in uses of the World Wide Web (WWW) recently has also benefited education, as many $\mathrm{K}-12$ schools are now regularly seeking information and resources for learning and teaching there. Schools themselves have been creating a presence on the Web. with over 5000 school-based Web sites registered worldwide as of November 1996 (Web66, 1996). But Web servers do not automatically make for educationally-useful resources. In fact, we believe that the majority of resources available on the WWW do not sufficiently meet the needs of students and teachers (Gordin et al., in press). It is interesting to note that the Web itself is one of the first major developments in computer and communication technology to make its presence felt at all levels of computer-using society, from school and work to home and community. The Web did not experience the "trickle down" that is present in so many other forms of new technology (Pea and Gomez, 1992). CoVis classrooms have been a part of the Web revolution at each step in its development, using Web resources since the early prototype versions of NCSA's Mosaic were available. The CoVis Project released its first Web server in early 1994.

Educational Needs of Learners and Teachers. Web servers that are designed with the needs of education in mind will have teaching and learning-cen- tered design, including special purpose "front-ends" to web resources, and supporting structures that help educational users match resources to teaching-learning needs. They need to support the following resources:

1. "Just in time" activities, materials, and resources to support classroom curricula

2. Facilities to support user authoring and browsing of commentaries on resources and how they may be applied in the classroom

3. Facilities for teachers, students, and other members of a learning community to establish connections to those who share common interests

4. Powerful facilities for searching and filtering the vast array of resources generally available on the Web

An educationally focused Web server will also include suggested or tried-and-true activity structures, and sample assessment rubrics to guide teachers in the integration of activity into daily classroom life. Educational Web servers must be designed to grow organically as the needs of their user population change and mature.

What Did We Develop? In response to these challenges, we developed the CoVis Geosciences Web Server, available on-line at http://www.covis nwu.edu/Geosciences/index.html. Introduced in the third year of the CoVis project, the design of this server is a direct result of ongoing conversations with teachers, students, geoscientists, and learning researchers about the resources they required to achieve our shared vision of science education. The Geosciences Web Server has at its core four components designed to address the needs of learners and teachers as enumerated above.

A Web section called "Activities" provides teachers with access to a growing number of curriculum units tied to the Geosciences. These units include land use management planning, water quality, soil science, global warming, and meteorological science. Each set of materials consists of sample activities for introducing students to the topic, pointers to relevant resources, access to listserv-based discussion groups for teachers, sample assessment rubrics and links to state and national standards, and suggestions for culminating activities. It is important to stress that these materials are not the on-line equivalent of "shrink-wrapped" curricula. These materials are intended to serve as jumping-off points for teachers, and thus all require local adjustments before being useful in particular classrooms. An important role of these shared activities is that they provide the necessary shared contexts for building interconnections between classrooms. The activities posted by CoVis are intended to be seeds for a large activities development process involving teachers. Teachers are encouraged to submit their own activities (via e-mail) and to submit their variations on the CoVis-developed activities. 
The "Teachers' Lounge" Web section provides links to materials about assessment, links to materials on the philosophy of teaching project-based science and related topics, and access to several resources designed to support teachers' daily work tasks. The first of these resources is a facility that allows teachers to create and annotate personal Web pages. We call these "link lists," and they are essentially a bookmark list created by teachers for use by their students. This facility does not require teacher's to have their own server, or to know HTML. Thus, it provides an entry point for the creation of content by teachers on the Web. A second resource available here is called the "CoVis Mentor Database," and it serves the function of an "intellectual ride board" to connect scientists who want to participate in student science projects to students and teachers who seek connections and advice from beyond the classroom (O'Neill et al., 1996).

A third Web section called "Resources" is a collection of on-line scientific visualization tools and links to other web servers with pertinent Geosciences information. The visualization tools (discussed in more detail in an earlier section) include a greenhouse effect visualizer, and a new Java-based interactive weather visualizer designed for CoVis by UIUC (Hall et al., 1996). Although we have not yet created powerful search engines to help teachers and students make sense of the broad and emerging range of on-line resources, we have established a collection of links to resources that we know about today.

A section called "Community News" provides the important function of a "town square" for teachers and students to share information about exciting events in their classrooms and schools. This is an important community-building component of the Geosciences Web Server. It provides a window for people to learn about what their colleagues are doing and recognizes excellence.

How Did It Work in Use? Resources like Web servers are often evaluated from a technology-oriented perspective-how many "hits" do they receive? On this dimension, CoVis resources score well, receiving thousands of hits each week from uses within our CoVis community of schools, but also from schools and other places around the world. From another perspective, we know that as of November 1996, over 2000 users and organizations have created Web pages linking to the CoVis home page (and we have no way of knowing how many others bookmark the site in their Web browsers). These CoVis Web links outside the CoVis hosts imply usefulness of these resources, but the statistics do not tell how they are being used. However, we believe that a more appropriate measure of success for educational Web services is the extent to which empirical observations indicate that they serve the needs of the community for which they are designed. In this regard, our results are more mixed.

On the positive side, teachers report that these services are valuable to them in their classrooms, and when we visit classrooms, we find that the CoVis home page has been set as the default, or that the school's own home page points prominently to ours. In a sense, our user community "votes with its feet," so resources that are not perceived as valuable receive much less use than those that are seen as essential. The pages dedicated to our curriculum and activities and the scientific visualizers are the most popular, and by translation, the most useful to our teachers at the moment. Newer resources such as the Mentor Database are still being tested, and we expect their popularity to grow as well.

A downside to the usefulness of our Web services is tied not to their design but rather to limitations associated with the Web in general. In particular, the network can become sluggish at peak times of use throughout the day. This means that the most graphically intensive resources, such as the visualization tools, are often not practical for use in classes. At the heart of the issue is that Internet resource availability is not guaranteed, and frequently network problems arise at precisely the moment when teachers were hoping to use them.

What Challenges Remain? Web servers-like textbooks and other media before them-must continue to evolve so as to be useful in teaching and learning contexts. The CoVis Geosciences WWW server provides an initial step in this direction. A growing number of Web-based applications must be developed that can be appropriated by teachers, and there is much work yet to be done to design Web resources that encourage sharing and make it easy for teachers to make their own insights, experiences, and resources available to a broader community of their peers and colleagues. Ongoing issues of bandwidth limitations must also be met before educational Web services are broadly useful.

\section{Conclusions}

In the past, technologists and marketers have considered education an "application" for their existing products and technologies rather than a driver of new products and services. Our point here has been that it is more productive to think of teaching and learning as a driver of technology development. Although this perspective has been rare in the past, the development of cyberspace is an opportunity to design new underlying features and applications motivated by the needs of teaching and learning rather than seeing education as just a reason to repurpose and repackage existing applications.

We used our experience in establishing and evolving the CoVis testbed to illustrate this point (see also Gomez et al., submitted). It is clear from our experience that we have had successes engaging teachers and children when we have understood their needs and created applications that fundamentally reflected teaching and learning contexts. In contrast, we have been least successful when we attempted to transfer, wholesale, an application or application-concept to schools without the necessary rethinking or fundamental reconceptualization. Our experience with Bellcore's Cruiser videoconferencing is a case in point. We believe that teachers, at least in part, shied away from videoconferencing because 
the form in which we presented it to them came directly from the world of white collar office work. In that world, for example, people often have private space in which to work. Personal video conferencing makes sense. In the class, private space is minimal. Therefore, informal video conferencing must be re-thought for the classroom.

On the other hand, we were more successful with the Collaboratory Notebook and scientific visualization applications because they were informed by the needs of learning communities from their earliest inception. In the case of the Collaboratory Notebook, we started with a general notion-collaborative hypermedia - and shaped the design of the application in iterations of classroom use and teachers' commentaries. In the case of the scientific visualization applications, tacit knowledge of the scientists who use such tools was made explicit and easier access to meaningful activities provided through the creation of custom visualization front-ends for specific domains of inquiry. We subsequently advanced the "fit" of scientific visualization as a tool for learning to the interests of teachers, and of learners. We did this in two ways: (1) by establishing, as teachers called for, CoVis Interschool Activities that set up a context of curriculum topics and activities to guide the uses of visualization, and (2) by reconceptualizing visualization as a medium for the classroom that emphasized students expressing their interests and beliefs through visualizations as well as interpreting the visualizations of scientists. Further, we added human geographic data sets (e.g., population) to address what we found to be preexisting student interests.

An essential requirement of our effort to create applications of cyberspace that reflect the needs of teaching and learning contexts has been evolving our view of what it means for technology to be integrally used in schools. Traditionally, the designers' goal is often thought about as one of getting technology "adopted." We see the adoption metaphor as fundamentally limited, a distorted one-way view of the route to integral technology use. The adoption perspective assumes that a technology comes packaged to a community. Shortcomings are viewed as problems with the adopters, not with the technology. A contrasting view is that technologies and other artifacts are "appropriated" by people rather than adopted (Pea, 1992). In this view technologies come to be used by individuals and communities based on a two-way process of "reciprocal evolution" (Allen, 1993). The user and designer each interpret the utility of an artifact. The artifact is then subject to cycles of iteration that reshape it based on the needs of the community of users and the designer's ongoing inventive responses to those needs (Greenbaum and Kyng, 1991; Schuler and Namioka, 1993).

The CoVis design goal is to create a tool suite shaped by a process of appropriation that includes the use of participatory design methods for refining the functionality and interface properties of new cyberspace applications coupled with intensive professional development activities for teachers as codesigners. If we are successful, teachers will innovate educational practices that are learner-centered. They will not see applications as simply being "delivered" to them for a prescribed use, but will invent uses in their local contexts that we cannot foresee.

Since we began the CoVis Project in 1992, our intentions of crafting a model collaboratory devoted to distributed science learning have led to current work with 43 schools in 11 states involving thousands of students. These schools are extraordinarily diverse in terms of socioeconomic profile, geography, and in bandwidth connectivity to the Internet. The primary challenge continues to be developing the practices and the tools of the collaboratory so that it is appropriated into teaching and learning practices, not simply "delivered" for use. In addition to the Collaboratory itself we hope to evolve design principles that will assist others in the creation of collaboratories that can be appropriated by other communities of practice.

\section{NOTES}

The CoVis Project has been funded by National Science Foundation Grants MDR-9253462 and MDR-9454729, Illinois Board of Higher Education Eisenhower grants, and our industrial partners Ameritech and Bellcore. We are also grateful for hardware and/or software contributions by Aldus, Apple Computer Farallon Computing, Sony Corporation, Spyglass, and Sun Microsystems. An earlier version of this chapter appeared in the 1995 Proceedings of the Internet Society. For additional information, check the World Wide Web address http://www.covis.nwu.edu.

\section{REFERENCES}

C. L. Alten, "Reciprocal evolution: A design and research strategy for the development and integration of basic research, design, and studies of work practices," in D. Schuler and A. Namioka, eds. Participatory Design. (Hillsdale, NJ: Lawrence Erlbaum Associates, 1993), pp. 239-253.

Bellcore Information Networking Research Laboratory, "The Touring Machine System," Communi cations of the Association for Computing Machinery 36(1)(1993):68-77.

K. W. Brodie, L. A. Carpenter, R. A. Earnshaw, J. R. Gallop. R. J. Hubbold, A. M. Mumford, C. D. Osland, and P. Quarendon, Scientific Visualization (Berlin: Springer-Verlag, 1992).

J. S. Brown, A. Collins, and P. Duguid, "Situated cognition and the culture of learning," Educational Researcher, $18(1)(1989): 32-42$.

V. G. Cerf, A. G. W. Cameron, J. Lederberg, C. T. Russell, B. R. Schatz, P. M. B. Shames, L. S. Sproull, R. A. Weller, W. A. Wulf, National Collaboratories: Applying hrormation Technology for Scientific Research (Washington, DC: National Academy Press, 1993).

A. Collins, J. S. Brown, and S. E. Newman, "Cognitive apprenticeship: Teaching the crafts of reading, writing, and mathematics," in L. B. Resnick, ed., Knowing, Learning, and Instruction: Essays in Honor of Robert Glaser (Hillsdale, NJ: Erlbaum, 1989), pp. 453-494.

L. Cuban, Teachers and Machines: The Classroom Use of Technology since 1920 (New York: Teachers College Press, 1986) 
D. C. Edelson and D. K. O'Neill, "The CoVis Collaboratory Notebook: Supporting collaborative scientific inquiry," in A. Best, ed., Proceedings of The 1994 National Educational Computing Conference (Eugene, OR: International Society for Technology in Education, 1994), pp. 146-152.

D. C. Edeison, D. K. O'Neill, L. M. Gomez, and L. D'Amico, "A Design for Effective Support of Inquiry and Collaboration," in J. L. Schnase and E. L. Cunnius, eds., Proceedings of CSCL '95: The First International Conference on Computer Support for Collaborative Learning. (Hillsdale. NJ: Erlbaum. 1995a).

D. C. Edelson, R. D. Pea, and L. Gomez, "Constructivism in the collaboratory," in B. G. Wilson, ed.. Constructivist Leaming Environments: Case Studies in Instructional Design (Englewood Cliffs, NJ: Educational Technology Publications, 1995b), pp. 15!-164.

R. S. Fish, R. E. Kraut, R. W. Root, and R. E. Rice, "Video as a technology for informal communication." Communications of the Association for Computing Machinery 36(1)(1993):48-61.

C. Fisher, D. C. Dwyer, and K. Yocam, eds., Education and Technology: Reflections on Computing in Classrooms (San Francisco, CA: Jossey-Bass Publishers, 1996).

B. J. Fishman, High-end high school communication: Tool use practices of students in a networked environment, Unpublished doctoral dissertation, Northwestern University, Evanston, IL (1996).

B. Fishman and L. D'Amico, "Which way will the wind blow? Networked computer too's for studying the weather," in T. Ottmann and I. Tomek, eds., Educational Multimedia and Hypermedia, 1994, Proceedings of Ed-Media'94 (Charlottesville, VA: AACE, 1994), pp. 209-216.

L. M. Gomez, B. Fishman, and J. Polman, "Media spaces and their application in K-12 and college leaming communities," Proceedings of the 1994 Conference on Computer-Human Interaction (Conference Companion) (New York: ACM Press, 1994). pp. 185-186.

L. M. Gomez, B. J. Fishman, and R. D. Pea, "The CoVis Project: Building a large scale science education testbed," Interactive Learning Environments (in press).

D. Gordin and R. D. Pea, "Prospects for scientific visualization as an educational technology," Journal of the Learning Sciences, 4(3)(1995):249-279.

D. N. Gordin, J. L. Poiman, and R. D. Pea, "The Climate Visualizer: Sense-making through scientific visualization," Journal of Science Education and Technology, 3(1994):203-226.

D. N. Gordin, D. C. Edelson, and R. D. Pea, "The Greenhouse Effect Visualizer: A tool for the science classroom," Proceedings of the Fourth American Meteorological Society Education Symposium, Dallas (1995).

D. N. Gordin, D. C. Edelson, and L. Gomez, "Scientific visualization as an interpretive and expressive medium," Proceedings of the International Conference on the Learning Sciences (Evanston, IL: Northwestern University, 1996a).

D. N. Gordin, D. C. Edelson, L. M. Gomez, M. Lento. and R. D. Pea, "Student conference on global warming: A collaborative network-supported ecologically hierarchic geosciences curriculum," Proceedings of the Fifth American Meteorological Society Education Symposium, Atlanta (1996b).

D. N. Gordin, D. C. Edelson, and R. D. Pea, Supporting students 'science inquiry through scientific visualization activities, Paper presented at the American Educational Research Association, New York (1996c).

D. Gordin, L. Gomez, R. D. Pea, and B. Fishman, "Using the World Wide Web to build learning communities in K-12," Journal of Computer-Mediated Communication 2(3)(1996).

3. Greenbaum and M. Kyng, eds., Design at work: Cooperative Design of Computer Systems (Hillsdale, NJ: Lawrence Erlbaum, 1991).

S. Hall, M. Ramamurthy, R. Wilhelmson, D. Plutchak, D. Wojtowicz, and M. Sridhar, The Weather Visualizer: A Java Tool for Imteractive Learning. Proceedings of the Education Initiatives Symposium, International Geoscience and Remote Sensing Symposium, Lincoln. NE (1996).
S. Heaviside, E. Farris, G. Malitz, and J. Carpenter, Advanced telecommunications in U.S. Public Elementary and Secondary Schools, 1995 (Fast Response Survey System NCES 96-854) (Washington, DC: National Center for Education Statistics, U.S. Department of Education, 1996).

J. Hawkins and R. D. Pea, "Tools for bridging everyday and scientific thinking," Journal for Research in Science Teaching, 24(4)(1987):291-307.

J. Lave and E. Wenger, Situated Learning: Legitimate Peripheral Participation (New York: Cambridge University Press, 1991).

J. Lederberg and K. Uncapher, eds., Towards a National Collaboratory: Report of an Invitational Workshop at the Rockefeller University, March 17-18, 1989 (Washington DC: National Science Foundation Directorate for Computer and Information Science, 1989).

M. C. Linn, N. B. Songer, and B. Eylon, "Shifts and convergences in science learning and instruction," in D. Berliner and R. Calfee, eds., Handbook of Educational Psychology (New York: Macmillan, 1995).

S. McGee, "Where is your data? A look at student projects in geoscience," Proceedings of the Fourth Symposium on Education at the 75th Annual Meeting of the American Meteorological Society (1995).

S. McGee, Designing curriculum based on science communities of practice, Unpublished doctoral dissertation, Northwestern University, Evanston, IL (1996).

S. McGee and R. D. Pea, "Cyclone in the classroom: Bringing the atmospheric sciences community in to the high school," in Proceedings of the Third American Meteorological Society Symposium on Education. 74th Annual Meeting of the AMS (Nashville TN: American Meteorological Society. 1994), pp. 23-26.

McKinsey \& Co., Connecting K-12 Schools to the Information Superhighway (Palo Alto, CA, 1995).

M. L. Markus, "Toward a 'critical mass' theory of interactive media: Universal access, interdependence, and diffusion," Commurication Research, 14(5)(1987):491-511,

B. Means, ed., Technology and Education Reform: The Reality behind the Promise (San Francisco, CA: Jossey Bass, 1994).

B. Nardi, ed., Context and Consciousness: Activity Theory and Human-Computer Interaction (Cambridge, MA: MIT Press, 1996)

National Center for Education Statistics, Advanced Telecommunications in U.S. Public Elementary and Secondary Schools, 1995 (Washington, DC: U.S. Department of Education, 1996) (http://www.ed.gov/Technology/starrpt.html)

National Research Council, The National Science Education Standards (Washington, DC: National Academy Press, 1996).

D. Norman, and S. Draper, eds., User-Centered System Design (Hillsdale, NJ: Lawrence Erlbaum, 1986).

D. K. O'Neill, D. C. Edelson, L. M. Gomez, and L. D'Amico, "Learning to Weave Collaborative Hypermedia into Classroom Practice," in J. L. Schnase and E. L. Cunnius, eds., Proceedings of CSCL '95: The First International Conference on Computer Support for Collaborative Learning (Hillsdale, NJ: Lawrence Erlbaum, 1995).

D. K. O'Neill, R. Wagner, and L. M. Gomez, "Online mentors: Experimenting in science class," Educational Leadership, 54(3)(1996):39-42.

S. Papert, The Children's Machine: Rethinking School in the Age of the Computer (New York, NY: Basic Books, 1994).

R. D. Pea, "Augmenting the discourse of learning with computer-based learning environments," in E. de Corte, M. Linn, and L. Verschaffel, eds., Computer-Based Learning Environments and Problem-Solving (NATO Series, subseries F: Computer and System Sciences) (Berlin: Springer-Verlag, 1992), pp. 313-343.

R. D. Pea, "The Collaborative Visualization Project," Communications of the Association for Computing Machinery, 36(5)(1993):60-63. 
R. D. Pea, "Seeing what we build together: Distributed multimedia learning environments for transformative communications," Journal of the Learning Sciences, 3(3)(1994):283-298.

R. D. Pea and L. M. Gomez, "Distributed multimedia leaming environments: Why and how?" Interactive Learning Environments, 2(2)(1992):73-109.

M. Ramamurthy. R. Wilhelmson. S. Hall, M. Sridhar, and J. Kemp. "Networked multimedia systems and collaborative visualization," in Proceedings of the Third Symposium on Education. 74th Annual Meeting of the American Meteorological Society (Nashville. TN: American Meteorological Socicly. 1994), pr, J30--133.

M. Ramanurthy, R. Wilhelmson, S. Hall, J. Plutchak, M. Sridhar, B. Fishman, D. Gordin, R. D. Pea. and L. M. Gomez, "CoVis Geosciences Web Server: An Internet-based resource for the K-12 community," Proceedings of the Fifth American Meteorological Society Education Symposium (1996).

R. W. Riley, M. M. Kunin, M. S. Smith, and L. G. Roberts, Getting America's Students Ready for the 2lst Century: Meeting the Technology Literacy Challenge-A Report to the Nation on Technology and Education (Washington, DC: U.S. Department of Education, 1996). (http://www. ed. gov/Technology/Plan/NatTechPlan/title.html)

R. Ruopp, S. Gal, B. Drayton, and M. Pfister, LabNet: Toward a Community of Practice (Hillsdale, NJ: Lawrence Erlbaum. 1993).

D. Schuler and A. Namioka, eds., Participatory Design: Principles and Practices. (Hillsdale, NJ: Lawrence Erlbaum, 1993).

N. B. Songer, "Knowledge construction through exchange and dialogue: A case study of Kids as Global Scientists," Journal of the Learning Sciences (1995).

K. Tobin, ed., The Practice of Constructivism in Science Education (Washington, DC: AAAS Press, 1993).

D. Tyack and L. Cuban, Tinkering Toward Utopia: A Century of Public School Reform. (Cambridge, MA: Harvard University Press, 1995).

U.S. Congress Office of Technology Assessment, Power On! New Tools for Teaching and Learning (Washington, DC: U.S. Office of Technology Assessment, 1987).

U.S. Congress Office of Technology Assessment. Teachers \& Technology: Making the Comection (OTA-EHR-616) (Washington. DC: U.S. Government Printing Office, 1995).

Web66. International WWW school registry [online document] (1996). Available: hitp://web66.coled.umn.edu/schools/stats/stats.html.

R. Wolff, and L. Yaeger, Visualization of Natural Phenomena (Berlin: Springer-Verlag, 1993). Includes CD-ROM. 